\title{
Acidentes por quedas em um grupo específico de idosos ${ }^{1}$
}

Accidental falls in an especific aged group

Accidentes por caídas en un grupo específico de ancianos

Alice Gabrielle de Sousa CostaI, Rozyane Cândido de SouzaII, Allyne Fortes Vitor ${ }^{\mathrm{III}}$, Thelma Leite de Araujo ${ }^{\mathrm{IV}}$

\section{RESUMO}

Este estudo objetivou conhecer a incidência do evento queda e identificar a presença de seus principais fatores de risco. Estudo exploratório, realizado de março a novembro/2009, com aplicação de um formulário sobre quedas em um grupo de idosos. Os dados foram analisados por cálculo de frequências, média e desvio-padrão. Participaram 62 idosos, 41,9\% relataram queda nos últimos seis meses, a maioria mulheres. Identificou-se ocorrência de agravos concomitantes: visão regular, audição boa, polifarmácia, IMC normal, forte força de preensão palmar e condições dos pés adequadas. Na maioria dos que caiu, o desequilíbrio foi apontado como principal motivo. A queda ocorreu mais no período da manhã, em local de piso áspero e seco, sem degraus, rampas ou tapetes, iluminação adequada e o tipo de calçado mais utilizado foi chinelo de borracha. Percebe-se a alta ocorrência das quedas na população idosa, fato que fundamenta a necessidade de avaliação das condições de risco envolvidas.

Descritores: Idoso; Acidentes por quedas; Enfermagem.

\section{ABSTRACT}

This study aimed to know the falls incidence and to identify its main risk factors. This is an exploratory study developed from March to November/2009, when was applied a form about falls in an elderly group. The data were analyzed by frequencies, mean and standard deviation. The participants were 62 aged and 41,9\% related falls occurrence at least six months, the majority was women. Was identified simultaneously health problems occurrence: regular vision, good audition, many medications use, normal BMI, strong power of palm hold and suitable feet conditions. Most fallers appointed less balance as the main reason. The fall occurred more in the morning, in rough and smooth floor, without steps, ramps or carpets, suitable illumination and the kind of shoes more used was rubber slipper. Perceives high falls occurrence at aged people that shows the necessity of evaluation about the risk conditions involved.

Descriptors: Aged; Accidental Falls; Nursing.

\section{RESUMEN}

Este estudio objetivó conocer la incidencia de caídas e identificar la presencia de sus factores de riesgo. Estudio exploratorio, desarrollado de marzo a noviembre/2009, con aplicación de un formulario en un grupo de mayores. Los datos fueron analizados por frecuencias, media y desvío estándar. Participaron 62 ancianos, 41,9\% relataron caídas en los últimos seis meses, la mayoría mujeres. Se identificó ocurrencia de quejas concomitantes: vista regular, buena audición, uso de muchas medicinas, IMC normal, fuerte fuerza de agarre palmar y condiciones de los pies adecuadas. La mayoría de los que cayeron, lo desequilibrio fue designado como principal razón. El evento ocurrió más por la mañana, en sitio con suelo áspero, seco, sin pasos, rampas ó alfombras, iluminación adecuada e lo calzado más utilizado fue la zapatilla de goma. Se percebe la alta ocurrencia de caídas en la población anciana, fundamentando la necesidad de evaluación de las condiciones de riesgo envueltas.

Descriptores: Anciano; Accidentes por Caídas; Enfermería.

\footnotetext{
${ }^{1}$ Artigo desenvolvido no Projeto Integrado Cuidado em Saúde Cardiovascular, financiado pelo CNPq (MCT/CNPq 14/2009 - Universal, Processo 472570/2009-9).

I Enfermeira, Mestre em Enfermagem,. Discente do Programa de Pós-Graduac ão em Enfermagem, nível Doutorado, Universidade Federal do Ceará (UFC). Fortaleza, CE, Brasil. E-mail: alice gabrielle@yahoo.com.br.

${ }^{\text {II }}$ Enfermeira. Enfermeira do Programa de Medicina Preventiva, UNIMED Fortaleza. Fortaleza, CE, Brasil. rozyane cs@hotmail.com.

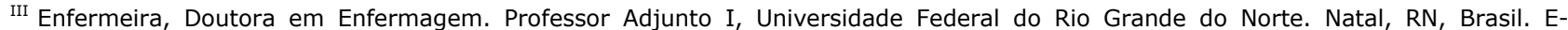
mail: allynefortes@yahoo.com.br.

IV Enfermeira, Doutora em Enfermagem. Professora Associada III, UFC. Fortaleza, CE, Brasil. E-mail: thelma@ufc.br.
} 


\section{INTRODUÇÃO}

A população brasileira, gradativamente, passa por mudança no cenário demográfico, diante do número de idosos que cresce em proporção considerável, com diminuição da mortalidade e consequente aumento de expectativa de vida dos brasileiros ${ }^{(1)}$. Com isso, questões dessa fase da vida assumem papel de destaque no desenvolvimento de políticas de promoção da saúde e prevenção de agravos.

Assim, as últimas décadas marcam-se pela expansão do contingente de idosos no país, correspondente, hoje, a mais de 18 milhões de pessoas, ou seja, quase $10 \%$ da população brasileira(2).

Os indivíduos, por sua vez, vivenciam alterações estruturais e funcionais produzidas pelo envelhecimento, entre as quais, se destacam as de mobilidade física: osteoporose, instabilidade postural, alteração de marcha, lentidão dos mecanismos de integração central, dificuldades visuais, auditivas e diminuição da força muscular ${ }^{(3)}$.

Em especial as alterações caracterizam-se como eventos incapacitantes, de incidência elevada, que são foco de preocupação social, pela geração não só de prejuízo físico e psicológico, mas também pelo aumento dos custos financeiros com os cuidados de saúde prestados $^{(1)}$.

Frente às mudanças, evidencia-se maior gravidade das consequências geradas pela ocorrência de quedas em idade avançada, em comparação com o público jovem. No idoso, a queda intensifica, ainda mais, a incapacidade física e emocional, levando-o, muitas vezes, a experimentar longos períodos de internação, reabilitação demorada, dependência posterior e risco considerável de morte ${ }^{(4)}$.

A queda é, então, uma importante alteração de saúde que pode ser definida como qualquer toque ao chão inesperadamente por qualquer parte do corpo do indivíduo, com exceção da sola dos pés ${ }^{(5)}$.

Este agravo adquire importância singular no cenário da saúde. No ano de 2007, por exemplo, as quedas representaram $41,58 \%$ de internações hospitalares por causas externas no país, atingindo, aproximadamente, 70.000 pessoas, maioria do sexo feminino $(46,2 \%)$ e com idade igual ou acima de 70 anos $(57,51 \%)^{(6)}$.

Os fatores de risco relacionados à queda podem ser multifatoriais, em condições intrínsecas e extrínsecas. Entende-se por fatores intrínsecos os relacionados ao indivíduo como idade, doenças e fatores psicológicos, enquanto os extrínsecos se referem aos comportamentos e atividades das pessoas idosas e ao ambiente físico ${ }^{(1)}$.
Nesse panorama, práticas saudáveis, como a realização de exercícios físicos, destacam-se em virtude dos inúmeros benefícios aos idosos, com melhoria da capacidade funcional, equilíbrio, força, coordenação e velocidade de movimento. São aspectos colaboradores ao aumento da segurança desses indivíduos, importantes para a prevenção de eventos incapacitantes ${ }^{(7)}$.

Incentivar a adoção dessas práticas preventivas é papel da equipe multiprofissional, indispensável ao atendimento desses indivíduos. Assim, é necessário pautar-se na integralidade do cuidado, em que o papel do enfermeiro assume dimensionamento ampliado, na lógica da vigilância da saúde quanto à promoção, prevenção, cura e reabilitação das condições de saúde ${ }^{(8)}$.

Nesta perspectiva, conhecer a incidência do evento e identificar seus fatores de risco na população idosa pode subsidiar a elaboração de estratégias de prevenção de quedas e conduzir as ações de programas de treinamento de profissionais da saúde, assim como políticas de saúde voltadas ao idoso.

Desse modo, o objetivo do estudo é identificar, em um grupo de idosos, a ocorrência de quedas e os fatores de risco que podem predispor o evento.

\section{MÉTODOS}

Trata-se de um estudo descritivo-exploratório, com abordagem quantitativa, realizado no período de março a novembro de 2009, em um complexo de saúde do idoso, na cidade de Fortaleza-Ceará. Os participantes foram idosos que frequentavam as reuniões semanais do complexo, as quais eram voltadas ao desenvolvimento de atividades lúdicas e de promoção da saúde.

A frequência média de participantes por reunião era de 70 idosos, que foram selecionados mediante amostragem por conveniência, em que todas as pessoas que atendessem aos critérios estabelecidos eram convidadas a participar da pesquisa. Assim, como critérios de inclusão, além de fornecer anuência e assinatura do Termo de Consentimento Livre e Esclarecido, os participantes deveriam ter idade igual ou superior a 60 anos e estar presente na reunião semanal do complexo de saúde no período da pesquisa. Foram excluídos os voluntários que apresentassem comprometimentos mentais caracterizados pela incapacidade de compreender e responder às questões do formulário, obtendo-se um total de 62 participantes.

O instrumento de coleta de dados foi construído com base em estudo anterior ${ }^{(9)}$ sobre quedas em idosos. Foi composto de questões de identificação e dados 
socioeconômicos, além de investigação sobre as variáveis condições intrínsecas, como percepção do estado de saúde, condições de visão e audição, uso de medicamentos, peso, altura e estado dos pés. Também investigou-se variáveis relativas à ocorrência de quedas nos últimos seis meses e, em caso positivo, buscou-se ainda caracterizar as condições ambientais ou extrínsecas do evento, tais como local de ocorrência, condições do piso e do calçado, iluminação e presença de objetos.

Para a coleta de dados antropométricos (peso e estatura), utilizou-se balança antropométrica com haste metálica, com capacidade de 150 quilogramas $(\mathrm{kg})$ e precisão de 100 gramas ( $\mathrm{g}$ ). As condições dos pés foram avaliadas por meio de métodos propedêuticos de inspeção e palpação no intuito de se identificar variáveis como deformidades, edema, unhas encravadas ou calosidades.

Para avaliação do condicionamento físico foi utilizado o instrumento proposto pela Rede de Pesquisa em Atenção Primária de Dartmouth/Organização Mundial das Escolas Nacionais, Academias e Associações Acadêmicas de Clínica Médica e Médicos da Família (COOP/WONCA $)^{(10)}$ composto por sete tópicos que avaliam o estado funcional do paciente, durante as duas últimas semanas na qual são investigadas as seguintes variáveis: forma física, sentimentos, atividades sociais, atividades cotidianas, mudança no estado de saúde, estado de saúde e dor, aspectos estes avaliados com base em figuras ilustrativas e escala de pontos que variam de um a cinco. As pontuações finais mais altas indicam piores capacidades funcionais. Ademais, utilizouse o Questionário Internacional de Atividade Física (IPAQ) $^{(11)}$ que permite estimar o tempo semanal gasto na realização de atividades físicas de intensidade moderada a vigorosa, e em diferentes contextos da vida.

Os dados foram compilados no programa Excel e analisados mediante cálculo de frequências percentuais e da medida de tendência central (média) e seu respectivo desvio-padrão, em seguida os dados foram organizados em tabelas, de acordo com as condições intrínsecas dos participantes que informaram ocorrência ou não de quedas, assim como as condições extrínsecas do ambiente onde as quedas ocorreram.

Ressalta-se que o estudo foi aprovado por Comitê de Ética em Pesquisa da Universidade Federal do Ceará, protocolo no 243/09, em atenção às recomendações da Resolução 196/96 do Conselho Nacional de Saúde, em pesquisas com seres humanos ${ }^{(12)}$. Os participantes foram orientados sobre os objetivos do estudo e assinaram o Termo de Consentimento Livre e Esclarecido.

\section{RESULTADOS}

Participaram do estudo 62 idosos, dos quais 26 $(41,9 \%)$ relataram episódio de quedas nos últimos seis meses. A maioria dos idosos era do sexo feminino, tanto no grupo com relato de quedas $(88,5 \%)$ quanto naquele em que o evento não foi mencionado $(66,6 \%)$, estes apresentaram média de idade ligeiramente superior (69,5 anos) em relação aos participantes com história de queda (67,9 anos).

O nível de escolaridade dos entrevistados, estimado em anos de estudo, foi de $7,7(+/-4,64)$ anos, em relação à ocupação, a maior parte $(83,9 \%)$ era de aposentados ou pensionistas, e a média da renda mensal foi de $1,4(+/-0,62)$ salários mínimos. As variáveis relativas às condições intrínsecas da ocorrência de quedas seguem apresentadas na Tabela 1. 
Tabela 1: Distribuição dos participantes que caíram e que não caíram segundo as condições intrínsecas envolvidas. Fortaleza, CE, 2009.

\begin{tabular}{|c|c|c|c|c|}
\hline \multirow{2}{*}{ Variáveis } & \multicolumn{2}{|c|}{ Idosos que caíram } & \multicolumn{2}{|c|}{ Idosos que não caíram } \\
\hline & $\mathbf{F A}^{1}$ & $\%$ & FA $^{1}$ & $\%$ \\
\hline Usa Medicamentos & 21 & 80,8 & 23 & 63,9 \\
\hline Possui alterações nos pés & 11 & 42,3 & 9 & 25,0 \\
\hline Pratica Esporte & 15 & 57,7 & 23 & 63,9 \\
\hline \multicolumn{5}{|l|}{ Estado de Saúde } \\
\hline Ótimo & 1 & 3,8 & 2 & 5,6 \\
\hline Bom & 8 & 30,8 & 20 & 55,5 \\
\hline Regular & 9 & 34,6 & 12 & 33,3 \\
\hline Má & 6 & 23,1 & 1 & 2,8 \\
\hline Péssimo & 2 & 7,7 & 1 & 2,8 \\
\hline Total & 26 & $100 \%$ & 36 & $100,0 \%$ \\
\hline \multicolumn{5}{|l|}{ Qualidade da visão } \\
\hline Quase cego / cego & 1 & 3,8 & 1 & 2,8 \\
\hline Ruim & 11 & 42,3 & 11 & 30,5 \\
\hline Regular & 10 & 38,5 & 11 & 30,5 \\
\hline Boa & 2 & 7,7 & 10 & 27,8 \\
\hline Excelente & 2 & 7,7 & 3 & 8,4 \\
\hline Total & 26 & $100,0 \%$ & 36 & $100,0 \%$ \\
\hline \multicolumn{5}{|l|}{ Qualidade da audição } \\
\hline Ruim & 3 & 11,5 & 3 & 8,3 \\
\hline Regular & 8 & 30,8 & 1 & 2,8 \\
\hline Boa & 10 & 38,5 & 27 & 75,0 \\
\hline Excelente & 5 & 19,2 & 5 & 13,9 \\
\hline Total & 26 & $100,0 \%$ & 36 & $100,0 \%$ \\
\hline \multicolumn{5}{|l|}{ IMC $^{2}$} \\
\hline Normal & 13 & 52,0 & 21 & 60,0 \\
\hline Sobrepeso & 5 & 20,0 & 13 & 37,1 \\
\hline Obeso & 7 & 28,0 & 1 & 2,9 \\
\hline Total & 25 & $100,0 \%$ & 35 & $100,0 \%$ \\
\hline \multicolumn{5}{|l|}{ IPAC $^{3}$} \\
\hline Baixo & 8 & 30,8 & 12 & 33,3 \\
\hline Moderado & 9 & 34,6 & 20 & 55,6 \\
\hline Alto & 9 & 34,6 & 4 & 11,1 \\
\hline \multirow[t]{2}{*}{ Total } & 26 & $100,0 \%$ & 36 & $100,0 \%$ \\
\hline & Média & DP $^{4}$ & Média & DP $^{4}$ \\
\hline Instrumento proposto por COOP / WONCA & 3,42 & 0,70 & 3,41 & 0,99 \\
\hline${ }^{1}$ Frequência Absoluta; & \multirow{2}{*}{\multicolumn{4}{|c|}{$\begin{array}{l}{ }^{3} \text { IPAC: Questionári } \\
{ }^{4} \text { Desvio-Padrão. }\end{array}$}} \\
\hline 2 Índice de massa corporal; & & & & \\
\hline
\end{tabular}

Identificou-se utilização de medicamentos pela maioria dos idosos, fato comum a essa população, destaca-se, contudo, que no grupo de idosos com relato de quedas, a utilização de medicamentos foi maior, com predominância dos anti-hipertensivos, hipoglicemiantes e psicotrópicos, os quais podem causar efeito hipotensor. Encontrou-se ainda o uso concomitante de duas ou mais classes de medicamentos, caracterizando a polifarmácia.
As alterações nos pés também foram observadas na maioria das pessoas que caíram, as quais relataram também menos prática esportiva, como dança, caminhada, hidroginástica e musculação leve. Estas práticas, por sua vez, eram incentivadas por instituições públicas com ações voltadas aos idosos e localizadas próximas às comunidades.

Quanto ao estado de saúde atual, as respostas se concentraram nas opções boa ou regular, mas chama 
atenção o número expressivo de idosos que informaram ocorrência de queda e afirmaram ter má saúde $(23,1 \%)$.

Com referência à capacidade visual, grande parte dos sujeitos mencionou condição visual ruim, mesmo com o uso de óculos e, sobre o estado de audição, obtido mediante a conversação durante a coleta de dados, a maioria dos entrevistados disse ter boa capacidade auditiva. Vale ressaltar que $11,5 \%$ dos indivíduos que caíram mencionaram possuir condição ruim de audição.

Em ambos os grupos, encontrou-se uma maioria de participantes com Índice de Massa Corporal adequado (normal), seguido por idosos com sobrepeso ou obesidade. Entre os participantes deste estudo que vivenciaram quedas, não houve nenhum com baixo peso, entretanto observou-se um percentual expressivo de idosos caracterizados como obesos.

Acerca da prática habitual de atividade avaliada pelo Questionário IPAC, com relação à população que não caiu, houve predominância de usuários em nível moderado de atividade. No concernente aos idosos que caíram, a prática de atividade física esteve proporcional em todos os níveis investigados. Sobre o condicionamento físico medido pelo instrumento proposto por COOP/WONCA, obteve-se média similar nos dois grupos. Identificou-se, todavia, maior desvio-padrão entre os indivíduos que negaram a ocorrência de quedas $( \pm 0,99)$.

Segundo constatado, $41,9 \%$ do total de idosos sofreram quedas nos últimos seis meses e, para a maioria, o evento não trouxe nenhum prejuízo. No entanto, para outra parcela menos expressiva, as consequências foram diversas, das quais dor generalizada foi a mais frequente nesses indivíduos. Vale ressaltar que a maior parte das vítimas ingeriu alguma medicação antes do evento, contudo, não houve relatos de consumo de bebidas alcoólicas nos dias de ocorrência das quedas.

As quedas ocorreram, majoritariamente, no período da manhã. As justificativas quanto à ocorrência relacionaram-se tanto a questões do ambiente, como condições fisiológicas intrínsecas, entre as mais citadas destacam-se desatenção e perda de equilíbrio, conforme demonstrado na Tabela 2. 
Tabela 2: Distribuição dos participantes do estudo que caíram de acordo com as informações extrínsecas. Fortaleza, CE, 2009.

\begin{tabular}{|c|c|c|}
\hline Variáveis & FA $^{1}$ & $\%$ \\
\hline \multicolumn{3}{|l|}{ Local do acidente } \\
\hline Via pública & 17 & $65,4 \%$ \\
\hline Cozinha & 4 & $15,4 \%$ \\
\hline Sala & 3 & $11,5 \%$ \\
\hline Banheiro & 2 & $7,7 \%$ \\
\hline Total & 26 & $100,0 \%$ \\
\hline \multicolumn{3}{|l|}{ Tipo de piso encontrado no local } \\
\hline Áspero & 14 & $53,8 \%$ \\
\hline Liso & 9 & $34,6 \%$ \\
\hline Outros & 3 & $11,5 \%$ \\
\hline Total & 26 & $100,0 \%$ \\
\hline \multicolumn{3}{|l|}{ Estado do piso } \\
\hline Seco & 21 & $80,8 \%$ \\
\hline Molhado & 4 & $15,4 \%$ \\
\hline Outros & 1 & $3,9 \%$ \\
\hline Total & 26 & $100,0 \%$ \\
\hline \multicolumn{3}{|l|}{ Tipo de calçado usado no dia da queda } \\
\hline Chinelo & 12 & $46,2 \%$ \\
\hline Sandália & 9 & $34,6 \%$ \\
\hline Sapato fechado & 3 & $11,5 \%$ \\
\hline Outros & 2 & $7,7 \%$ \\
\hline Total & 26 & $100,0 \%$ \\
\hline \multicolumn{3}{|l|}{ Tipo de solado do calçado } \\
\hline Borracha & 18 & $69,2 \%$ \\
\hline Couro & 6 & $23,0 \%$ \\
\hline Outros & 1 & $3,9 \%$ \\
\hline Total & 26 & $100,0 \%$ \\
\hline \multicolumn{3}{|l|}{ Iluminação do local } \\
\hline Clara & 23 & $88,5 \%$ \\
\hline Escura & 3 & $11,5 \%$ \\
\hline Total & 26 & $100,0 \%$ \\
\hline Presença de degraus & 5 & $19,2 \%$ \\
\hline Presença de rampas & 1 & $3,9 \%$ \\
\hline Presença de tapetes & 2 & $7,7 \%$ \\
\hline Presença de animais ou objetos & 2 & $7,7 \%$ \\
\hline
\end{tabular}

${ }^{1}$ Frequência Absoluta.

Com relação ao ambiente, a maioria das quedas foi observada em via pública, com presença de piso áspero e seco. Do mesmo modo, a maior parte desses acidentes ocorreu na presença de degraus, rampas, tapetes, animais ou objetos que pudessem dificultar a passagem das vítimas. O calçado mais usado durante as quedas eram chinelos com solado de borracha.

O ambiente, em $88,5 \%$ dos casos, foi considerado pelo participante claro o suficiente a ponto de não interferir na capacidade de visualização dos objetos ou superfícies.

\section{DISCUSSÃo}

As quedas e suas consequências se fazem presentes em todas as épocas da vida, no entanto, são especialmente denotadas como um problema de saúde quando na idade mais avançada, haja vista a 
possibilidade de lesões graves e à possibilidade de impactos psicológicos resultantes desse evento.

Neste estudo, houve predominância de população feminina, principalmente entre idosos que sofreram quedas. Outra pesquisa concorda com a maior frequência de quedas entre pessoas do sexo feminino devido à maior fragilidade osteomuscular e à osteoartrose, fato relacionado à redução do estrógeno a partir dos 40 anos de idade, assim como, maior vínculo com as atividades domésticas ${ }^{(13)}$.

No estudo ora realizado, grande parte dos participantes vítimas de quedas apresentaram baixa escolaridade, achado semelhante em outra investigação na qual pessoas com maior instrução apresentaram maiores preocupações com saúde e com as estruturas responsáveis pelo controle postural, assim como com a capacidade de recuperação, fato que pode diminuir, de forma direta ou indireta, a susceptibilidade às quedas ${ }^{(14)}$.

Ademais, a percepção dos idosos sobre seu estado de saúde influencia significativamente a ocorrência de quedas. Segundo pesquisas, quanto pior a percepção sobre a saúde por parte do idoso, mais passiva é sua atitude frente às enfermidades. Isto pode estar relacionado com a aceleração da deterioração física, aumento da probabilidade de apresentar algum distúrbio no equilíbrio e potencialização do risco de sofrer queda $^{(14)}$.

Conforme estudos sobre envelhecimento, alterações como perda de massa muscular e óssea e alterações no equilíbrio, peculiares à senescência, aumentam o risco de cair. Tais alterações podem ser minimizadas com a prática regular de atividade física devido à melhora da mobilidade física e da estabilidade postural(15-16). Os resultados do presente estudo estão em concordância com esses achados, pois do total de entrevistados, mais da metade se exercitava regularmente e de forma moderada.

Ainda sobre o fato, pesquisas comprovam a relação diretamente proporcional entre a prática regular de atividade física e a melhora da capacidade funcional do idoso, de modo que se torna fundamental a prevenção de quedas ${ }^{(7,17)}$.

Quase a totalidade dos idosos referiu boa acuidade auditiva, a maior parte fazia uso de óculos, embora a melhora da visão com uso de óculos era apenas parcial. Outros trabalhos apontam que as alterações sensoriais que diminuem a capacidade de enxergar podem levar à ocorrência de vertigens. Além disso, os déficits visuais ou óculos inadequados, relacionados com a pouca iluminação ambiental, podem predispor escorregões e tropeços. A diminuição da acuidade auditiva, como afirmam outras pesquisas, pode acarretar redução das "pistas" ambientais, bem como prejudicar o controle postural $^{(9,18)}$.

A grande quantidade de medicamentos utilizada pelos participantes representa, ainda, outro fator preocupante, porquanto os efeitos deletérios da interação medicamentosa são mais acentuados nessa faixa etária.

Conforme estudos, a polifarmácia predispõe o idoso a efeitos adversos potencializados, como sonolência, fadiga, tonturas, hipotensão ortostática, vertigem, urgência urinária $e$, por causarem diminuição do tempo de reação, dificuldades no equilíbrio e no caminhar, arritmias e danos a um estado de alerta cognitivo, podem conduzir a um risco maior de $\operatorname{cair}^{(9,14)}$.

A maior parcela dos idosos entrevistados usava várias drogas concomitantemente, e mais da metade fez uso de medicamentos no dia da queda. Entre esses últimos, os anti-hipertensivos, seguidos dos hipoglicemiantes e psicotrópicos foram os mais referidos.

O estado nutricional também está relacionado com o maior ou menor risco de queda de idosos. Nesse âmbito, a atenção volta-se para os extremos de classificação do Índice de Massa Corporal: o baixo peso e a obesidade ${ }^{(9)}$. Entre os participantes acometidos por quedas, não houve nenhum com baixo peso, a maioria apresentava uma classificação de IMC dentro dos parâmetros de normalidade e um percentual expressivo de idosos foi caracterizado como obeso.

De acordo com outras investigações sobre saúde do idoso, os obesos costumam apresentar estrutura física desigual e maior, e assim, comprometer o equilíbrio, muitas vezes já alterado em consequência do processo de envelhecimento, o que acentua o risco de o idoso cair $^{(2,5,18)}$.

Ressalta-se, além disso, o número expressivo de indivíduos com problemas podológicos, possivelmente influenciadores da marcha. Neste âmbito, estudos reforçam a importância da manutenção de cuidados adequados com os pés, pois se trata de estratégia fundamental na prevenção de déficits de marcha em idosos, notadamente nos portadores de neuropatias periféricas ${ }^{(9)}$.

A causa das quedas ainda pode estar relacionada a fatores extrínsecos como o ambiente em que elas ocorreram. Neste estudo, as quedas ocorreram, majoritariamente, fora do ambiente doméstico, em ruas, avenidas ou calçadas. O relato de quedas em locais fora da casa também foi mais percebido em outra pesquisa, 
que verificou ainda a existência de calçadas irregulares no momento em que os idosos estavam caminhando como a principal causa de quedas destes ${ }^{(19)}$. Dos relatos de quedas dentro do domicílio, a cozinha foi o compartimento mais citado, pois, como referido anteriormente, a maioria dos idosos entrevistados era do sexo feminino, em que mais da metade eram donas de casa, ou auxiliavam nas atividades domésticas. Esses resultados contrariam, em parte, os encontrados em outros estudos, no quais a ocorrência de quedas concentra-se, sobretudo, no banheiro ${ }^{(9,13,18)}$.

Com relação ao período de ocorrência, as quedas aconteceram, em maior número, pela manhã e poucos foram os relatos de quedas à noite ou na madrugada. Esse resultado contraria a os achados de outro estudo de que os idosos caem mais durante a noite, quando levantam para tomar água ou para ir ao banheiro(9).

Quanto ao tipo de piso referido, o áspero foi o mais destacado, e contrasta com outros estudos nos quais as quedas foram resultantes de pisos escorregadios. Com relação às condições de iluminação, a maior parte dos acidentes ocorreu em ambiente claro, semelhante ao identificado em outra pesquisa no Brasil( $^{(9)}$ mas divergindo de um estudo realizado fora do país ${ }^{(17)}$.

Os chinelos com solado de borracha foram os mais usados por idosos durante as quedas. Pesquisas descrevem o sapato adequado para evitar quedas, que deve ser fechado para idosos, além de possuir solado antiderrapante, com suporte reforçado no calcanhar, de salto baixo, com meia-sola mais fina que o calcanhar, sendo flexível e firme, provido de mecanismo de amarração e sem pontos de tensão(13). Nesse âmbito, é essencial que o enfermeiro atente-se para esta variável e seja capaz de orientar e aconselhar os idosos quanto à compra de sapatos, considerando os requisitos básicos em detrimento das condições socioeconômicas do indivíduo.

$\mathrm{Na}$ maioria dos locais onde as quedas ocorreram, não havia degraus, rampas, corrimão, tapetes, animais ou objetos que pudessem dificultar a mobilidade ou a circulação dos idosos. Esses resultados foram semelhantes aos de outro estudo(9).

Destacam-se ainda os prejuízos ocasionados pelas quedas como dores generalizadas pelo corpo, fraturas de membros e dificuldade de deambulação. Os prejuízos de uma queda, além do desconforto físico, interferem drasticamente na realização das atividades básicas de vida dos idosos, pois, diante de uma autonomia reduzida, eles passam a depender de terceiros para o suprimento de suas necessidades. Dessa forma, vale ressaltar o alto índice de dependência física apresentado por população idosa, identificando-se, assim, predomínio de fatores de risco intrínsecos ${ }^{(20)}$.

Ademais, muitos idosos passam a ficar em estado de alerta excessivo, amedrontados, temendo novas quedas. Com isso, seus estilos de vida modificam-se e as atividades, muitas vezes rotineiras, são abandonadas $^{(15,17)}$.

O medo de cair, por exemplo, traz consigo alterações como perda de autonomia e independência para as atividades de vida diária, diminuição das atividades pessoais e sociais, além de sentimentos de fragilidade e insegurança(18).

Os idosos tiveram, ainda, a oportunidade de expor as justificativas para a queda vivenciada. Dentre as causas apresentadas, destacam-se a falta de equilíbrio e de atenção. Os fatores ambientais, no entanto, também estiveram presentes e merecem destaque por serem os mais facilmente modificáveis.

Prevenir a ocorrência de quedas ou minimizar as suas consequências é de fundamental importância, pois desta forma, é possível reduzir custos para o setor saúde. Portanto, estratégias de modificação ambiental somadas aos cuidados individuais voltados às alterações intrínsecas são de extremo valor para a promoção da saúde do idoso ${ }^{(7,14)}$.

Diante do exposto, percebe-se o quão relevante é a abordagem da ocorrência de quedas em pessoas de faixa etária mais elevada, visto ser um fenômeno de alta frequência nesta população. Sendo assim, a busca de fatores de risco entre idosos se faz necessária e deve ser reforçada entre cuidadores e profissionais de saúde, com vistas a uma maior redução da incidência de quedas.

\section{CONCLUSÃO}

Percebe-se que as quedas são eventos reais na vida dos idosos e traz a eles consequências, muitas vezes, irreparáveis. São eventos danosos não só às vítimas como também àqueles que convivem com o idoso. Além disso, as quedas representam ônus para o setor saúde e devem ser encaradas, definitivamente como um problema de saúde pública.

Nesse âmbito, conhecer o perfil da população atingida por este evento é importante, no intuito de melhor direcionar as ações de prevenção do profissional da saúde. Assim, como percebido no estudo em questão, as idosas de baixa escolaridade e nível social são aquelas que mais devem ser focadas. Destaca-se ainda que a prática de atividade física pode funcionar como um fator 
protetor para quedas, ressaltando a necessidade do incentivo na continuidade ou mesmo início de tal prática.

Percebe-se que as variáveis intrínsecas envolvidas com a queda são as mais percebidas no grupo de idosos, tais como mobilidade física prejudicada, problemas com equilíbrio e marcha ou alterações podológicas estão diretamente envolvidas com o aumento no risco de quedas e devem ser continuamente avaliadas com vistas à prevenir o agravo.

No entanto, os fatores de risco extrínsecos são os mais facilmente modificáveis. As quedas ocorreram em número maior nos ambientes públicos, como ruas e avenidas no momento em que os idosos caminhavam. Percebe-se, então, que as irregularidades arquitetônicas como calçadas desniveladas podem estar diretamente relacionadas ao aumento do risco de quedas, fato que suscita a necessidade de políticas públicas mais rígidas no intuito de sanar este problema.

Nesse sentido, são de extrema importância medidas de segurança ambiental, com o objetivo de minimizar o risco de romper a integridade física dessa população.
Outra medida imprescindível refere-se à modificação dos hábitos de vida e inserção gradativa de atividades físicas regulares, visto serem fundamentais na recuperação da capacidade funcional, do equilíbrio e da força muscular dos idosos, pois oferecem maior segurança e os tornam menos susceptíveis às quedas.

Um programa ou, até mesmo, estratégias bem direcionadas e eficientes de inserção de exercícios físicos na rotina dos idosos também podem aprimorar a capacidade física do indivíduo e melhorar a resistência cardiovascular, força, flexibilidade e equilíbrio.

Vale ressaltar ainda, a necessidade de acompanhamento periódico do estado de saúde dessas pessoas, com o intuito de controlar de forma mais eficaz as doenças crônicas, e assim, reduzir o risco de agravos maiores. Ademais, destaca-se a importância da avaliação dos medicamentos prescritos e o reconhecimento dos riscos da polifarmácia, uma vez que esses fatores estão diretamente associados à ocorrência de quedas entre idosos.

\section{REFERÊNCIAS}

1. Secretária de Atenção à Saúde, Ministério da Saúde. Envelhecimentos e saúde da pessoa idosa [Internet]. Brasília: Ministério da Saúde; 2006 [cited 2011 set 30]. Available from: http://dab.saude.gov.br/docs/publicacoes/cadernos_ab/abcad1 9.pdf.

2. Machado TR, Oliveira CJ, Costa FBC, Araujo TL. Avaliação da presença de risco para queda em idosos. Rev. Eletr. Enf. [Internet]. 2009 [cited 2011 set 30];11(1):32-8. Available from: http://www.fen.ufg.br/revista/v11/n1/pdf/v11n1a04.pdf.

3. Instituto Brasileiro de Geografia e Estatística. Perfil dos Idosos Responsáveis pelos Domicílios [Internet]. Brasília: Ministério do Planejamento, Orçamento e Gestão (BR); 2002 jul 25 [cited 2011 set 30]. Available from: http://www.ibge.gov.br/home/presidencia/noticias/250720 02pidoso.shtm.

4. Biazin DT, Rodrigues RAP. Perfil dos idosos que sofreram trauma em Londrina - Paraná. Rev Esc Enferm USP [Internet]. 2009 [cited 2011 set 30];43(3):602-8. Available from: http://www.scielo.br/pdf/reeusp/v43n3/a15v43n3.pdf.

5. Wada N, Sohmiya M, Shimizu T, Okamoto K, Shirakura K. Clinical analysis of risk factors for falls in home-living stroke patients using functional evaluation tools. Arch Phys Med Rehabil. 2007;88(12):1601-5.

6. DATASUS [Internet]. Brasília: Ministério da Saúde (BR) [cited 2011 set 30]. Indicadores de morbidade e fatores de risco Brasil - 2007. D.14 Proporção de internações hospitalares (SUS) por causas externas. Available from: http://tabnet.datasus.gov.br/cgi/deftohtm.exe?idb2007/d1 4.def.

7. Mazo GZ, Liposck DB, Ananda C, Prevê D. Condições de saúde, incidência de quedas e nível de atividade física dos idosos. Rev. Bras. Fisioter. [Internet]. 2007 [cited 2011 set 30];11(6):43742. from: http://www.scielo.br/pdf/rbfis/v11n6/v11n6a04.pdf.

8. Marin MJS, Cacílio LCO, Rodrigues LCR, Ricci FA, Druzian S. Diagnósticos de enfermagem de idosas carentes de um Programa de Saúde da Família (PSF). Esc. Anna Nery [Internet]. 2008 [cited 2011 set 30];12(2):278-84. Available from: http://www.scielo.br/pdf/ean/v12n2/v12n2a12.pdf.

9. Lojudice DC. Quedas em idosos institucionalizados: ocorrência e fatores associados [dissertation]. Ribeirão Preto: Faculdade de Medicina de Ribeirão Preto/USP; 2005.

10. Tudela LL, Ferrer AR. Evaluation in the clinic of health-related quality of life: the COOP/WONCA cartoons. Aten Primaria. $2002 ; 29(6): 378-84$.

11. Matsudo S, Araújo T, Matsudo V, Andrade D, Andrade E, Oliveira $C$ et al. Questionário Internacional de Atividade Física (IPAQ): estudo de validade e reprodutibilidade no Brasil. Rev. bras. ativ. fís. Saúde. 2001;6(2):5-18.

12. Ministério da Saúde; Conselho Nacional de Saúde. Resolução No 196/96 - Normas regulamentadoras de pesquisa envolvendo seres humanos. Brasília (Brasil): Ministério da Saúde; 1996.

13. Menant JC, Steele JR, Menz HB, Munro BJ, Lord SR. Optimizing footwear for older people at risk of falls. J Rehabil Res Dev. 2008;45(8):1167-81.

14. Maciel ACC, Guerra RO. Prevalência e fatores associados ao déficit de equilíbrio em idosos. Rev. bras. ciênc. mov [Internet]. 2005 [cited 2011 set 30];13(1):37-44. Available from: http://portalrevistas.ucb.br/index.php/RBCM/article/view/610/6 $\underline{22}$.

15. Guimarães JMN, Fariinatti PTV. Análise descritiva de variáveis teoricamente associadas ao risco de quedas em mulheres idosas. Rev Bras Med Esporte [Internet]. 2005 [cited 2011 set 30];11(5):299-305. Available from: http://www.scielo.br/pdf/rbme/v11n5/27593.pdf.

16. Siqueira FV, Facchini LA, Piccini RX, Tomasi E, Thumé E, Silveira DS et al. Prevalência de quedas em idosos e fatores associados. Rev Saude Publica [Internet]. 2007 [cited 2011 set 30];41(5):749-

56. http://www.scielosp.org/pdf/rsp/v41n5/6188.pdf.

17. Swann J. Fall prevention is everyone's responsibility. Nurs Res Care. 2008;10(6):294-8.

18. Rodrigues IG, Ruscalleda RMI. Satisfação com a vida e senso de autoeficácia para quedas em idosos. Rev. Soc. Bras. Clín. Méd. $2009 ; 7(6): 413-7$. 
19. Beck AP, Antes DL, Meurer ST, Benedetti TRB, Lopes MA. Fatores associados às quedas entre idosos praticantes de atividades físicas. Texto Contexto Enferm [Internet]. 2011 [cited 2011 set 30];20(2):280-6. Available from: http://www.scielo.br/pdf/tce/v20n2/a09v20n2.pdf.

20. Costa AGS, Oliveira ARS, Moreira RP, Cavalcante TF, Araujo TL. Identificação do risco de quedas em idosos após acidente vascular encefálico. Esc. Anna Nery [Internet]. 2010 [cited 2011 set 30];14(4):684-9. Available from: http://www.scielo.br/pdf/ean/v14n4/v14n4a05.pdf.

Artigo recebido em 10.05.2011.

Aprovado para publicação em 05.09.2011.

Artigo publicado em 30.09.2011. 